\title{
Extended Function Analysis of Urban Planning and Design Based on Automatic Extraction Algorithm of Closed Area Boundary
}

\author{
Xifeng Mi \\ Jiaozuo Normal College, Jiaozuo 454000, China \\ Correspondence should be addressed to Xifeng Mi; mixifeng@jzsz.edu.cn
}

Received 17 November 2021; Revised 13 December 2021; Accepted 24 December 2021; Published 13 January 2022

Academic Editor: Qiangyi Li

Copyright (c) 2022 Xifeng Mi. This is an open access article distributed under the Creative Commons Attribution License, which permits unrestricted use, distribution, and reproduction in any medium, provided the original work is properly cited.

\begin{abstract}
With the continuous development of social economy, the expansion of cities often leads to the disorderly utilization of land resources and even waste. In view of these limitations and requirements, this paper introduces the automatic extraction algorithm of closed area boundary, combs the requirements of urban boundary extraction involved in urban planning and design, and uses the technology of geospatial analysis to carry out spatial analysis practice from three angles, so as to realize the expansion of functional analysis of urban planning and design and improve the efficiency and rationality of urban planning. The simulation results show that the automatic extraction algorithm of closed area boundary is effective and can support the functional analysis of urban planning and design expansion.
\end{abstract}

\section{Introduction}

With the continuous development of social economy, urbanization is an important trend, but disorderly urbanization will not only cause the waste of urban land resources, but also destroy the ecological environment to a certain extent $[1,2]$. Therefore, many industry experts have put forward the concept of combining design with nature; that is, reasonable design and planning are needed to transform nature rather than destroy nature $[3,4]$. The development of Internet information technology has innovated the means of urban planning and design, so that more schemes can be used for two-dimensional, three-dimensional, and other multiangle reference. Geographic information science (GIS) is an interdisciplinary subject of geographic science and computer science. Its main feature is the powerful spatial analysis function. Therefore, for urban planning, the powerful spatial analysis function is helpful for design to avoid unnecessary loss or expenditure $[5,6]$. Since 1990, with the increasing maturity of spatial processing technology and the popularization of computer mapping, GIS technology has been widely used in the field of urban planning. It can graphically express spatial data and spatially analyze attribute data on a certain basis. The powerful spatial analysis function of GIS technology provides conditions for urban planning and design $[7,8]$.

AutoCAD software is one of the most popular vector drawing tools in the world and is also widely used in many fields $[9,10]$. For CAD images, closed areas are usually required to store attributes as work units. For example, entities such as flower beds and roads in cities need to be expressed by closed areas $[10,11]$. However, it is worth noting that for scattered and disordered graphics, how to effectively extract the boundary of closed area is the premise to improve planning, data processing, and data processing $[12,13]$. According to the research results of existing industry scholars, the boundary extraction of grid graphics can be carried out through mature algorithms, but extracting the boundary of vector images still cannot ensure the corresponding automatic extraction efficiency due to the topology and complex composition of vector data $[14,15]$.

However, it should be noted that for urban planning and design, it is very important to extract the closed boundary effectively and quickly. This paper introduces the algorithm 
of automatic extraction of closed area boundary, combs the relevant businesses of urban planning and design, carries out spatial analysis from three angles from the perspective of geospatial technology, and realizes urban planning and design, in order to build corresponding expansion functions and improve the efficiency and effect of urban planning and design.

\section{GIS Spatial Analysis Function}

GIS can be understood as geographic information system or geographic information science. Its essence is a new technical method. According to the characteristics of space, spatial data can be collected, stored, managed, processed, mined, analyzed, and visualized, which can be realized by calculation tools. Its characteristic essence is a kind of spatial analysis. Compared with CAD system, geographic information science has powerful spatial analysis function. At the same time, its visualization can be displayed in two-dimensional or three-dimensional, static or moving ways. For urban planning, it can invert the effect after planning, including the application of corresponding daylighting, flood damage, emergency, etc.

The main technical process of spatial analysis of general geographic information science is shown in Figure 1.

\section{Automatic Extraction Algorithm of Closed Region Boundary}

The corresponding vector graphics obtained by ordinary measurement can be composed of $\mathrm{N}$ line segments, which are further closed to form several regions. Therefore, the next problem to be solved is how to automatically and reasonably extract the boundary of the closed area in order to complete the extraction of the corresponding closed boundary $[16,17]$.

\subsection{Triangulation}

3.1.1. Basic Principles. As for the closed area of complex polygons, it is to use triangles to deal with problems. The specific steps are as follows: firstly, the corresponding complex graphics are divided into several triangles. On this basis, the corresponding fuzzy processing is carried out by computer to simplify the complex problems and realize the application of triangle dissection.

Initialize the corresponding settings. Suppose that the polygon has $n$ endpoints, where $n$ exceeds 3 , and a certain line segment is the diagonal of $\mathrm{P}$. The diagonal can divide the complex graph into left and right parts. Gradually increase the nonintersecting diagonal of the polygon, and iterate continuously until the interior of the polygon is divided into triangles (Figure 2).

For any complex polygon divided, triangulation is carried out to realize the orderly division of polygons; that is, all impassable triangles are spliced with common edges to realize the orderly combination of coverage and polygons. The process of extracting corresponding vector polygon by triangulation method is shown in Figure 3.

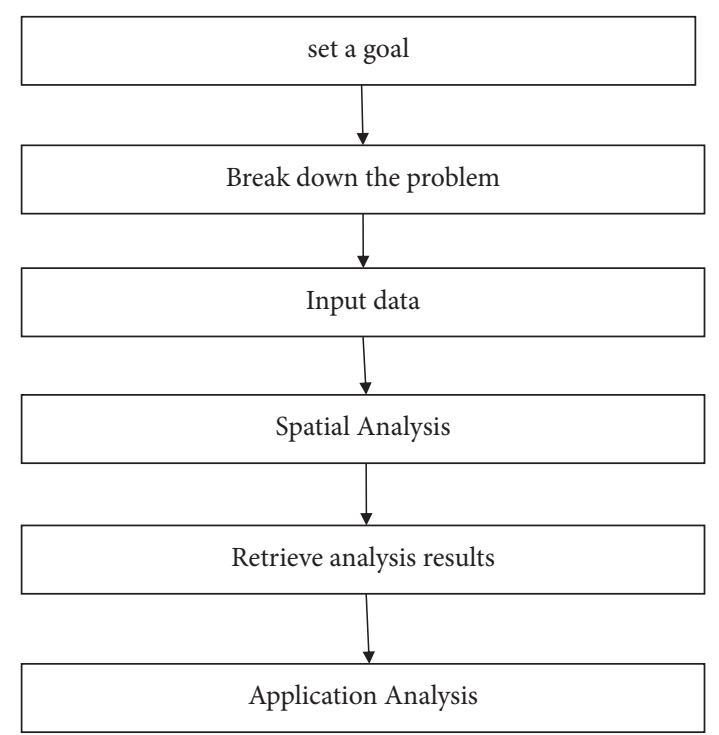

FIGURE 1: main steps and technical flow chart of spatial analysis.

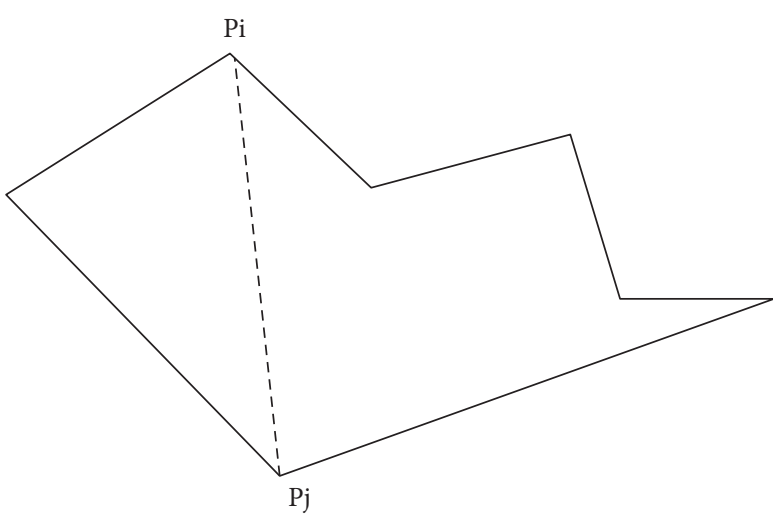

Figure 2: Schematic diagram of triangulation method.

\subsubsection{Algorithm Implementation Steps}

(1) Graphics Preprocessing.

(1) For the decomposition of polylines, firstly, select the corresponding vector graphics for processing, decompose the polylines of vector graphics, and obtain the polyline set of two points.

(2) Effective deletion of duplicate polylines. On the basis of the first step, filter and distinguish the duplicate polylines. If the corresponding parameters of the two polylines are opposite to each other, it can be determined that the two polylines are duplicate and can be deleted.

(3) Interpolate polylines. The calculation is performed between two points of the polyline. If the position of the focus complements the fixed point of the polyline, the insertion point is required.

(4) Explode the polyline again. There may be some polylines in C1. In step C, new vertices are inserted. Decompose these polylines to obtain a new two-point polyline set $\mathrm{C}$. 


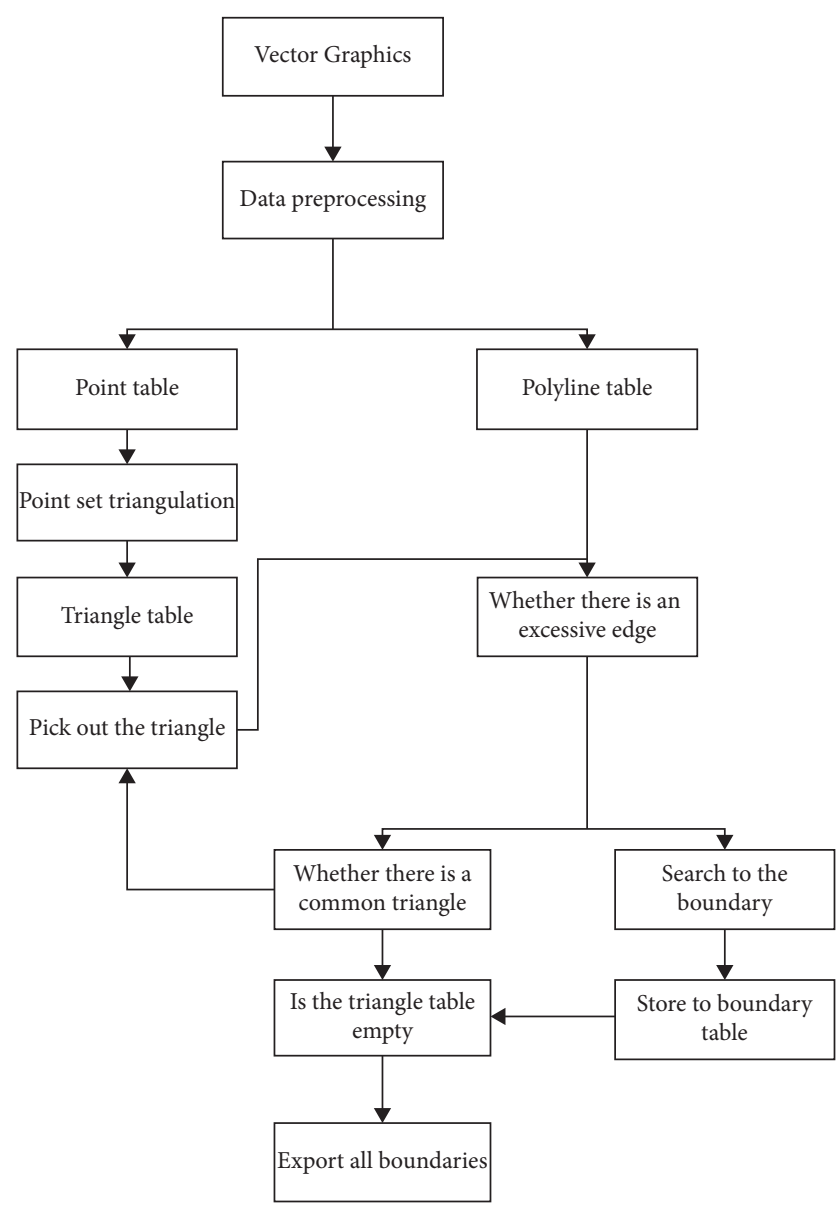

FIGURE 3: extraction of vector graphics boundary by triangulation method.

(5) Delete duplicate polylines again to further filter the polylines.

(6) Delete a dangling polyline. Remove the hanging polyline of the corresponding polyline.

(2) Create Polyline Table. Collect and sort out the polylines to realize the starting point, key point, convex point, etc. of the corresponding polylines.

(3) Create Point Table. Create a point table CP to record the vertices of all polylines in $\mathrm{C} 2$.

(4) Create Triangle Table. Triangulate the point set in the point table CP to obtain irregular triangulation (TIN). At this time, the polygonal triangulation of each closed area is also completed. Create a triangle table CT to record the three sides of each triangle in the triangulation.

(5) Search the Minimum Closed Region Boundary by Growth Method. Create a boundary table to store the extracted boundary information of the minimum closed area.

(6) Output Boundary. For each output boundary, the output of the boundary is realized.

\subsection{Area Method}

3.2.1. Basic Principles. For the region method, it is a basic graph, which uses the corresponding closed objects to create the closed area. The specific types of creation can be a straight line segment, broken line segment, arc segment, ellipse, spline segment, etc.

For regions, it can realize three functions: (1) testing the closure of graphics: the closed regions can be transformed into a complete graphics; if the graphics are not closed, they cannot be transformed directly; (2) realizing the corresponding parameter calculation of the region, such as the center of gravity and area of the region; (3) creating the corresponding three-dimensional model: if the plane graphics are transformed into regions, the lifting of threedimensional graphics and the formation of three-dimensional effect can be realized.

The corresponding code algorithm is used to realize the interface to create the corresponding area. Secondly, the face is effectively decomposed to realize the effective splicing and end-to-end connection of the object set of points, so as to form the minimum closed boundary of vector graphics $[17,18]$.

\subsubsection{Algorithm Implementation Steps}

(1) Graphics Preprocessing. Before the implementation of region method, complex graphics need to be preprocessed effectively.

(2) Create Regions. First, perform the static function CreateFromCurves of region class to effectively create regions. The specific definitions are as follows: public static DBObjectCollection CreateFromCurves (DBObjectCollection curveSegments).

(3) Explode Regions. On this basis, the effective decomposition of regions can be realized, and the corresponding set of regions can be obtained.

(4) Export Boundary. The curve set obtained in step (3) is spliced to obtain a minimum closed region boundary.

3.3. Model Interpretation. For urban planning and design, transportation is one of the important considerations. The passenger transport hub is an important node connecting the inside and outside and can also realize the effective development of the functional area of urban activities. In addition, the hub area exists as a node, which is the node of the traffic network and an important node of the city. On the one hand, it can realize the traffic attribute of the traffic hub; on the other hand, it can reflect the functional attribute of the traffic hub.

For the node and field model, it can realize the description of five scenarios, in which the two sides of the diagonal line segment in the middle are relatively balanced, indicating that the value of the node and field model is equal. According to the corresponding price, it can be divided into 
three types. Among them, the pressure area at the top shows that the intensity of traffic and various urban activities is at the maximum, as shown in Figure 4.

3.4. Change of Flow. In the process of urban planning, when a new railway passenger transport hub is built in the city, the total flow of the additional urban transportation system will change, and the mode of internal and external traffic conversion will change accordingly.

In urban planning and design, if a new railway passenger transport hub is built, it will further guide the rapid growth of external traffic and realize the increase of passenger flow. On the one hand, it will lead to more passengers concentrated in the new railway hub and improve the total share of urban external traffic; on the other hand, the increase of urban transportation facilities will further improve the positioning and transportation capacity of urban transportation.

First, according to the requirements of multiple inputs and single outputs, the neuron model is used, and the cell body is used for processing according to the corresponding external signals transmitted to the next neuron. The specific neuron model is shown in Figure 5.

According to the corresponding planning, establish the corresponding planning route neuron; each neuron is set as a planning node, and the planning motivation influence factor causes corresponding fluctuations, as shown in Figure 6.

On the basis of Figure 6, the corresponding planning one-way neural network model is established, as shown in Figure 7.

3.5. Field Change. With the continuous arrival and development of urban planning, the connection between the functions of various scales of the city will be further improved. Therefore, for those who have been engaged in railway travel for a long time, the time value will be effectively improved. Such demand and pressure make the transportation hub very important in urban planning and design.

3.5.1. Matrix of Decision Criterion Layer. Firstly, the matrix of the criterion layer is constructed, as shown in the following formula:

$$
A_{s}=A\left(a_{i j}\right)=\left(\begin{array}{cccc}
1 & a_{12} & a_{13} & a_{14} \\
a_{21} & 1 & a_{23} & a_{24} \\
a_{31} & a_{32} & 1 & a_{34} \\
a_{41} & a_{42} & a_{43} & 1
\end{array}\right] .
$$

The calculation formula of benefit type parameters is shown as follows:

$$
b_{i, m}=\frac{y_{i, m}-y_{i, m}^{\min }+1}{y_{i, m}^{\max }-y_{i, m}^{\min }+1} .
$$

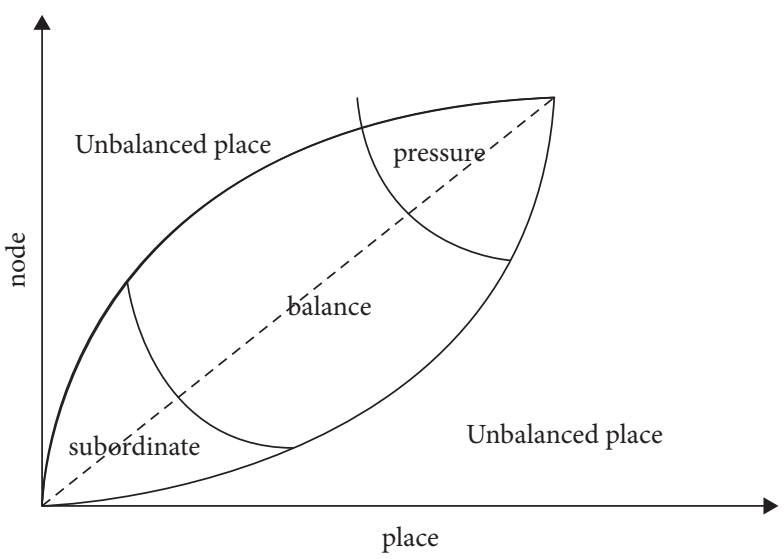

FIgUre 4: Node site model.

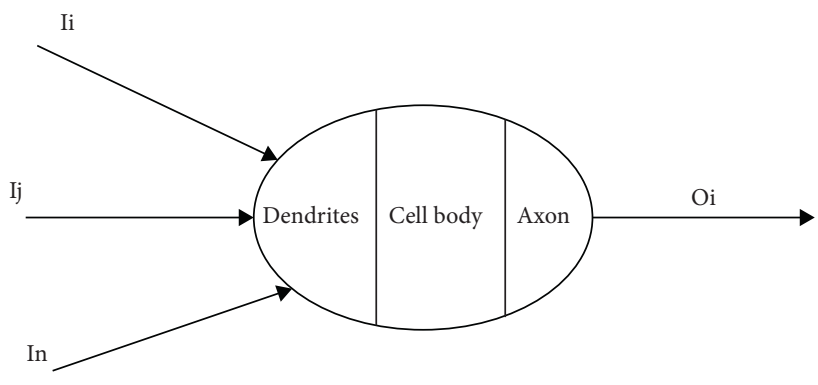

FIgURE 5: MP neuron model.

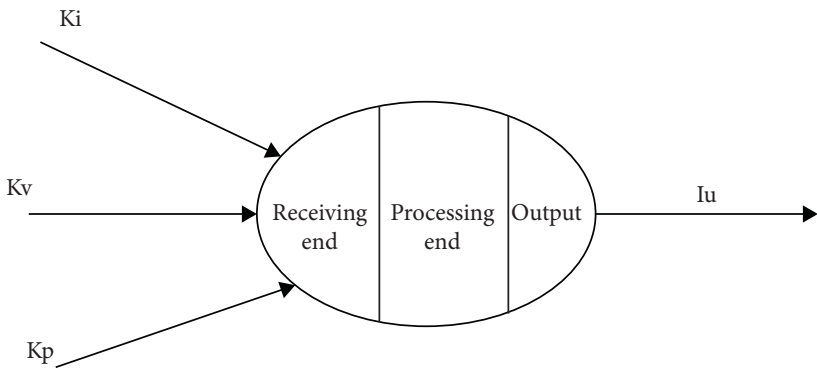

FIgURE 6: Neuron model of route.

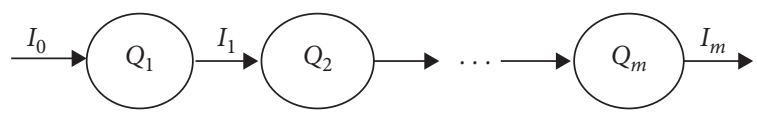

FIGURE 7: Route unidirectional neural chain model.

The calculation formula of cost type parameters is shown as follows:

$$
b_{i, m}=\frac{y_{i, m}^{\max }-y_{i, m}+1}{y_{i, m}^{\max }-y_{i, m}^{\min }+1} .
$$

For formulas (2) and (3), the main purpose is to normalize and quantify. The elements of the judgment matrix in the criterion layer are calculated as follows:

$$
a_{i j}=\frac{c_{i}}{c_{j}}=\frac{\sum_{m} b_{i, m}}{\sum_{m} b_{j, m}} .
$$


3.5.2. Determination of Scheme Level Judgment Matrix. Calculate the dimensionless value according to formula (3) and formula (4), as follows:

$$
A_{p, i}=\left[\begin{array}{cc}
1 & \frac{b_{i, 1} h}{b_{i, 2}} \\
\frac{b_{i, 2}}{\left(b_{i, 1} h\right)} & 1
\end{array}\right], \quad i=1,2,3,4
$$

Firstly, calculate the maximum value of the corresponding eigenvector of the matrix, and sort it in a single level; then, the sorting matrix can be calculated by the following formula:

$$
W_{k}=\left[\xi_{1}, \xi_{2}, \ldots, \xi_{N}\right]
$$

Calculate the maximum element value in the sorting matrix according to formula (7), as shown in the following formula:

$$
W=W_{k} W_{k-1} \ldots W_{2} W_{1} .
$$

The expansion model mainly starts from the overall development level of the urban transportation system and urban spatial structure and describes the development trends of two types of changes and three types of areas.

The first change refers to the reconstruction of traffic flow. The construction of the new hub relieves the external traffic flow of the old hub. At the same time, the construction of urban rail transit and the development of public transportation have improved the internal traffic relief capacity of the old hub. The new development is expected to unfold in this context.

The second change refers to the reorganization of the function of the place. During the period of regional integration, the construction of multiple centers on the periphery of megacities is inseparable from the development of existing urban centers. New urban rail transit links the existing urban centers with new hub areas. Urban centers (unbalanced places) that lack the support of traffic nodes and are densely developed can gain node functions, and their place functions can also be transmitted to new peripheral hub areas through new links.

The development trends of the three types of areas are as follows: (1) Existing railway passenger transport hubs have the characteristics of high node value and low place value, that is, unbalanced nodes. With the transformation and expansion of the hub system, the value of the place will be improved to return to balance. (2) Existing urban centers have the characteristics of high place value and low node value, that is, unbalanced places. For example, some places are affected by the construction of urban subway, regional express line, railway passenger dedicated line, and other transportation systems. They can improve the node function by improving accessibility and ease the place function to the outside of the city. (3) The changes in unbalanced nodes and unbalanced locations will affect newly built railway passenger transport hubs and obtain the development opportunities brought about by the passenger flow demand and unbalanced locations of existing railway passenger transport hubs. The location and node value of newly built railway passenger transport hubs are expected to be doubleenhanced.

The railway passenger transport hub system and the urban center system of the megacities are an organic whole. The development of the three types of regions is always in a process of dynamic change, and they influence each other and are inseparable. If the development of the surrounding areas of the hub aims to move toward balance, it needs the cooperation of other areas, and it also needs to be guided and supported by a broader planning vision and transportation space policy.

\section{GIS Technology and Urban Planning}

\subsection{GIS Technology Meets the Needs of Urban Planning and} Design. The continuous development of social science and technology can effectively realize spatial analysis, especially for modern urban planning and design. On the one hand, it is necessary to consider the actual positioning and development needs of the city. On the other hand, it is necessary to examine the surrounding environment in real time to realize the harmonious coexistence of man and nature. In the process of traditional urban planning and design, vector data is often stored through CAD for point, line, and surface classes. However, it should be noted that CAD data only has graphics and lacks corresponding attribute data, which brings subsequent application problems, such as the inability to query regions, especially attribute queries, in the process of browsing and editing, There will be problems such as the inability to recognize the data. GIS technology can effectively realize urban planning and meet the analysis of modern urban planning and design.

4.2. GIS Technology Can Improve the Rationality and Scientificity of Urban Planning. Because GIS has powerful spatial analysis function, it can realize the processing, mining, and management of spatial data, and the processing and analysis of various schemes in the process of urban planning and design, and it can realize the design analysis of urban planning and design expansion function. While the basic data is updated, the expansion function can still be used effectively for a long time. On the one hand, it can save costs, that is, the universality of extended functions; on the other hand, it can improve work efficiency and carry out batch operation.

Modern urban planning and design constitute an important standard to measure the quality of a city's development. The core of the development of a region is the development of the city. The progress and development of the city are promoted by the cooperation of the human beings and nature in the region. The planning and design of a city require a variety of complex spatial data and attribute data as support. However, these are cumbersome and complex. The data is constantly updated and changed within a certain period of time. Traditional urban planning and design cannot achieve accurate processing and analysis 
when faced with large-scale data. This is mainly due to the lack of advanced data analysis and processing tools, which leads to the data analysis being too rough to meet the scientific requirements. Traditional urban planning and design mostly focus on qualitative analysis, and the development opinions and strategies put forward are relatively subjective, mixed with many perceptual factors, resulting in relatively lagging planning data analysis results, and in addition the materials that planners rely on when formulating urban development plans are not sufficient, and various factors will ultimately affect the future development of the city.

With the increasing maturity of data analysis technology, the emergence and introduction of ArcGIS software have brought a new idea to the field of urban planning and design, accelerated the transformation of the thinking mode of urban planning and design, and led the direction of urban planning and design to a certain extent. It took a big turn. ArcGIS software has great advantages such as fast updating of data and intuitive spatial analysis. It can manage and analyze large-scale data scientifically and reasonably, which is helpful for the transformation from static to dynamic urban planning and design. At the same time, ArcGIS software technology has enriched the urban planning and design methods on a certain basis and made up for the single defect of the traditional urban planning and design methods. The application of ArcGIS software in modern urban planning and design has been paid more and more attention to. Its spatial analysis technology has been fully integrated into the entire process of urban planning and design; whether it is data collection or data processing, whether it is detailed or regional planning, whether it is static or dynamic simulation, GIS spatial analysis functions all play an irreplaceable role in urban planning and design, adding assistance to the planning and design of modern cities.

\section{Algorithm Verification}

5.1. Verification Example. The basement mapping in urban planning and design, for example, in the corresponding CAD drawing, can be composed of different multiple line segments, but only multiple line segments, rather than area attributes. The automatic extraction algorithm of closed region boundary and area is obviously better than the traditional human-computer interaction method. The accuracy of the method is shown in Figure 8:

5.2. Analysis of Validation Results. It can be seen from the results in Figure 8 that the automatic extraction algorithm of closed area boundary is effective and has high accuracy. Although the accuracy of traditional human-computer interaction is also high, it is still lower than the corresponding algorithm proposed in this paper. At the same time, in terms of processing speed and time-consuming, the algorithm proposed in this paper still takes a short time, but the processing time of other methods is also relatively short. Therefore, in different application scenarios and requirements, it is necessary to select an appropriate boundary extraction method.

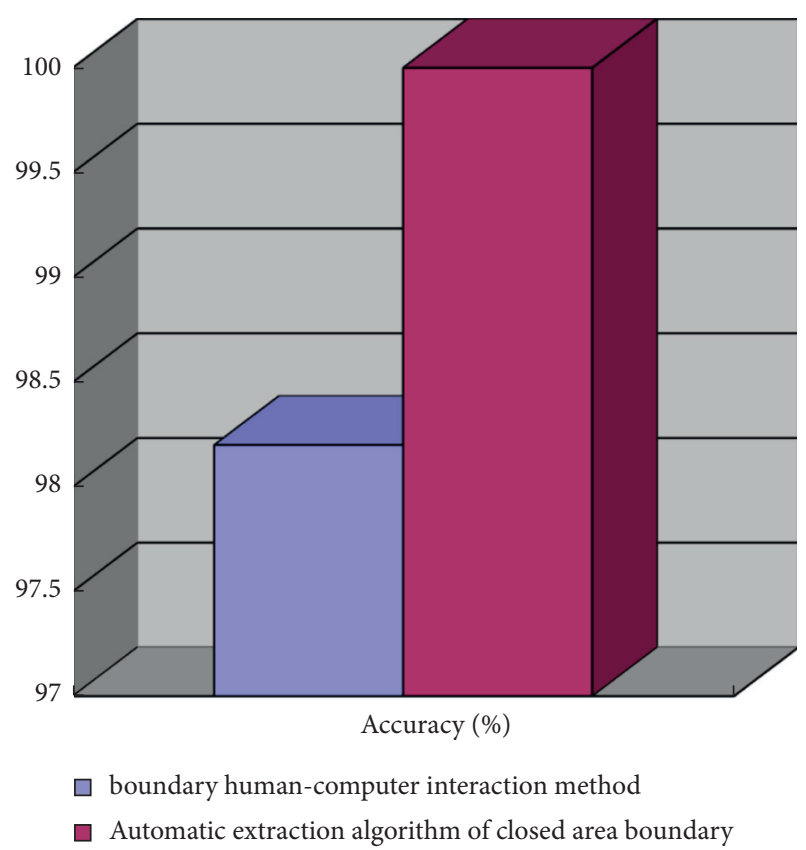

FIgURE 8: Comparison of accuracy of boundary extraction methods.

From the perspective of time consumption, the time the algorithm takes to automatically extract the boundary of the enclosed area is only about $1 / 33$ of that taken by the "boundary" human-computer interaction method, and the human-computer interaction method may cause longer consumption due to fatigue caused by simple repeated operations. However, by using the triangulation method and the area method executed by the program to automatically extract the boundary, the time consumption for the same workload is almost the same, and the operation efficiency is very high.

From the perspective of accuracy, the "boundary" human-computer interaction method requires the operator to click on the map more than 300 times, and there may be errors and omissions in the configuration. However, the triangulation method and the automatic extraction algorithm of the closed area boundary only need one operation to select the polyline to be processed, the operation is simple, and the accuracy of the execution result is high. During the test, it was also found that when calling the "boundary" command to obtain the boundary, if there are dense and small-sized turning lines in the graph, the generated boundary may miss some turning points, but triangulation and area methods are used. There is no error to deal with this situation.

The comparison between the triangulation method and the closed area boundary automatic extraction algorithm also shows that each has its own advantages and disadvantages. In terms of processing speed, the automatic extraction algorithm of the closed area boundary is even better. In the process of using programming language to realize the algorithm, the automatic extraction algorithm of the closed area boundary is also more intuitive and simple. However, AutoCAD has very strict requirements on graphics objects 
when creating regions. Some very subtle graphical differences that are almost indistinguishable by the human eye may cause region creation to fail. Therefore, the appropriate boundary extraction method should be selected according to different application scenarios and requirements.

In modern urban planning and design, urban road alignment design is one of the most important links. To a certain extent, the design and optimization of urban road alignment are closely related to the topographical features of the city, the current road alignment of the city, the arrangement and layout of buildings around the road, and the level of planned roads. In order to make the planned road alignment scientific and reasonable, it is necessary to accurately measure the road slope in combination with advanced GIS technology, and use the relevant theories and experience of urban road planning to make the planned road level reasonable, occupy less agricultural land, and be in line with the road. The surrounding buildings are arranged in a coordinated direction.

\subsection{Simulating the Terrain of the Planning Area and Opti-} mizing the Road Alignment. In modern urban planning and design, urban road alignment design is the most important link. To a certain extent, urban road alignment design and optimization are closely related to the urban topographic and geomorphic characteristics, the current urban road alignment, the arrangement and layout of buildings around the road, and the planned road grade.

\section{Conclusions}

With the continuous development of the city, orderly and effective urban planning and design are extremely important. In view of these needs and the existing status quo defects, based on the closed area boundary automatic extraction algorithm, this paper further combs the urban boundary extraction business process involved in urban planning and design and realizes the practice in urban planning and design from three angles based on the function of spatial analysis, in order to improve the efficiency of urban planning and design. The simulation results show that the automatic extraction algorithm of closed area boundary is effective and can further improve the depth and breadth of urban planning and design.

\section{Data Availability}

The labeled datasets used to support the findings of this study are available from the author upon request.

\section{Conflicts of Interest}

The author declares that there are no conflicts of interest.

\section{Acknowledgments}

This study was sponsored by Science and Technology Planning Project of Henan Province, China (No. 212400410223).

\section{References}

[1] S. Prestamburgo, F. Sgroi, and C. Zanin, "Anthropic space and design for all. New knowledge paths for urban planning strategies," The Science of the Total Environment, vol. 663, no. 1, pp. 944-949, 2019.

[2] A. Sharifi and A. R. Khavarian-Garmsir, "The COVID-19 pandemic: impacts on cities and major lessons for urban planning, design, and management," The Science of the Total Environment, vol. 749, no. 3, pp. 142-146, 2020.

[3] M. Pineda-Pinto, N. Frantzeskaki, and C. A. Nygaard, "The potential of nature-based solutions to deliver ecologically just cities: lessons for research and urban planning from a systematic literature review," Ambio, vol. 4, no. 5, pp. 1-16, 2021.

[4] S. Cinderby, A. de Bruin, H. Cambridge, C. Muhoza, and A. Ngabirano, "Transforming urban planning processes and outcomes through creative methods," Ambio, vol. 50, no. 5, pp. 1018-1034, 2021.

[5] V. Maquil, "Towards understanding the design space of tangible user interfaces for collaborative urban planning," Interacting with Computers, vol. 28, no. 3, pp. 332-351, 2015.

[6] M. M. Azari, F. Rosas, and S. Pollin, "Cellular connectivity for UAVs: network modeling, performance analysis, and design guidelines," IEEE Transactions on Wireless Communications, vol. 4, no. 5, pp. 3366-3381, 2019.

[7] E. Martinez, D. Perez, V. Gavani, and S. Kenny, "Introduction," Journal of Materials Research, vol. 33, no. 7, pp. 773-776, 2018.

[8] L. A. Saketkoo, "Successful trial design and planning in systemic sclerosis: does it take a village?" Journal of Rheumatology, vol. 45, no. 3, pp. 297-299, 2018.

[9] J. Maria Jebamalai, K. Marlein, J. Laverge, L. Vandevelde, and M. van den Broek, "An automated GIS-based planning and design tool for district heating: scenarios for a Dutch city," Energy, vol. 183, no. 15, pp. 487-496, 2019.

[10] J. A. Rodríguez Algeciras, H. Coch, G. De la Paz Pérez, M. Chaos Yeras, and A. Matzarakis, "Human thermal comfort conditions and urban planning in hot-humid climates-The case of Cuba," International Journal of Biometeorology, vol. 60, no. 8, pp. 1151-1164, 2016.

[11] P. Fontaine and S. Minner, "A dynamic discrete network design problem for maintenance planning in traffic networks," Annals of Operations Research, vol. 253, no. 2, pp. 1-8, 2016.

[12] H. Tang, T. Zhang, X. Liu, and Y. Jiang, "Novel method for the design of radiant floor cooling systems through homogenizing spatial solar radiation distribution," Solar Energy, vol. 170, no. 8, pp. 885-895, 2018.

[13] P. Y. Liew, W. L. Theo, and S. Alwi, "Total Site Heat Integration planning and design for industrial, urban and renewable systems," Renewable and Sustainable Energy Reviews, vol. 4, no. 6, pp. 136-140, 2016.

[14] B. Giles-Corti, A. Vernez-Moudon, and R. Reis, "Series Urban design, transport, and health 1 City planning and population health: a global challenge," The Lancet, vol. 6736, no. 16, pp. 300-316, 2016.

[15] J. Algeciras and A. Matzarakis, "Quantification of thermal bioclimate for the management of urban design in Mediterranean climate of Barcelona, Spain," International Journal of Biometeorology, vol. 60, no. 8, pp. 1-10, 2015.

[16] "Participatory systems approaches for urban and peri-urban agriculture planning: the role of system dynamics and spatial group model building," Agricultural Systems, vol. 160, pp. 110-123, 2018. 
[17] T. Norman, "Operational research in hospital planning and design," Journal of the Operational Research Society, vol. 8, no. 3, pp. 149-157, 2017.

[18] J. Zhen, G. Huang, W. Li, C. Wu, and Z. Liu, “An optimization model design for energy systems planning and management under considering air pollution control in Tangshan City, China," Journal of Process Control, vol. 47, no. 4, pp. 58-77, 2016. 